\title{
Iron refractory or Iron Resistant Iron Deficiency Anemia in Adult Celiac Disease Resolves with a Gluten-free Diet
}

\author{
Hugh James Freeman* \\ Department of Medicine (Gastroenterology), University of British Columbia, Vancouver, Canada \\ *Corresponding author: hugfree@shaw.ca
}

\begin{abstract}
Iron deficiency anemia is a common presentation of adult celiac disease. In a retrospective review of over 200 adults with celiac disease complicated by iron deficiency anemia, 7 patients were selected that were treated either with oral iron supplements with a limited hematologic response or unable to tolerate oral iron. Subsequently, treatment with a strict gluten free diet alone was provided. In all, resolution of the anemia resulted without a need for concomitant oral iron supplements. Another patient with marked fatigue and exercise intolerance was treated with intravenous iron, but the iron deficiency anemia only partially improved. After a strict gluten-free diet, her iron deficiency anemia resolved completely. Clinical experience in these celiac patients indicates that the key element in the treatment of iron deficiency anemia is a positive intestinal mucosal response to a gluten-free diet. In celiac patients with iron deficiency anemia, particularly with a limited hematologic response, intolerance or side effects to iron supplements, treatment solely with a gluten-free diet may be a reasonable option. Iron deficiency anemia in celiac disease is heterogeneous and may have multiple causes. Although duodenal mucosal disease is a critical factor, other factors may alter the regulation of iron homeostasis in this setting, including altered erythropoiesis due to the underlying chronic intestinal inflammatory process.
\end{abstract}

Keywords: adult celiac disease, iron deficiency anemia, iron refractory anemia, gluten-free diet

Cite This Article: Hugh James Freeman, "Iron refractory or Iron Resistant Iron Deficiency Anemia in Adult Celiac Disease Resolves with a Gluten-free Diet." International Journal of Celiac Disease, vol. 6, no. 1 (2018): 26-29. doi: 10.12691/ijcd-6-1-2.

\section{Introduction}

Iron deficiency anemia is a common presenting clinical feature for adult celiac disease, an immune-mediated enteropathy that primarily affects the duodenal mucosa and extends distally into the rest of the small intestine [1]. Clinical findings, particularly with extensive small intestinal disease, usually include chronic diarrhea and weight loss. In some, however, iron deficiency may occur alone in the absence of any other clinical feature typical of celiac disease. In part, this may be due to impaired iron uptake from the duodenum in celiac disease.

In the present cases here, iron deficiency anemia was present but, in these cases, referral was prompted because of iron deficiency anemia and the apparent failure to respond to iron administration. In these cases, studies to exclude an underlying cause were pursued, including celiac disease. Here, however, iron refractory or resistant iron deficiency anemia was shown to respond completely to a gluten-free diet alone without supplemental iron treatment.

\section{Patients and Methods}

Records from over 200 patients with biopsy-defined adult celiac disease were reviewed. Patients with iron deficiency anemia were examined, and for this evaluation, only patients initially treated with adequate amounts of iron and a documented failure of the anemia to resolve or improve ("iron refractory" or "iron resistant", respectively) were included. Patients with a concomitant cause for anemia (eg., folate depletion, vitamin B12 deficiency) or a separate gynecologic or gastrointestinal cause (eg. colonic neoplasia) were excluded. Most patients had serological studies and further duodenal biopsies on a gluten-free diet to ensure mucosal healing.

\section{Case Reports}

Case 1. A 40-yr-old Romanian-born female with intermittent diarrhea, constipation and abdominal pain was referred for evaluation in May 2007. She reported a 15 year history of iron deficiency anemia (with hemoglobin levels ranging from 95 to 105 ), treated continuously with oral ferrous sulphate $325 \mathrm{mg}$ tid. Family history revealed a deceased brother with insulin-dependent diabetes and her mother had anemia. Physical examination was normal, weight $57 \mathrm{~kg}$. Fecal occult blood negative. Laboratory studies confirmed anemia with a hemoglobin of $105 \mathrm{~g}$ per $\mathrm{L}$ (normal, 115 to 155 ) and MCV $80 \mathrm{fl}$ (normal, 82 to 98). A serum ferritin was 6 ug per L (normal, 20 to 300). A red 
cell folate was 946 mol per L (normal, 630 to 1790) and a serum vitamin B12 was 264 pmol per L (normal, 150 to 600). Other hematologic parameters (white cells, platelets) were normal as well as an INR, serum calcium, glucose, magnesium, albumin, TSH and liver chemistry tests. Immunoglobulin quantitation was normal (IgA, IgG, IgM) with a tissue transglutaminase (tTG) IgA antibody of 120 units (normal, less than 20). Abdominal ultrasound was normal with no gallstones. Colonoscopy and biopsies were normal. Upper GI endoscopy revealed scalloped duodenal mucosal folds and biopsies showed severe architectural changes with villous atrophy and crypt hyperplasia (i.e., Marsh 3), typical of untreated celiac disease. As oral iron was hypothesized to have contributed to her abdominal symptoms (diarrhea, constipation, abdominal pain), she was treated only with a gluten-free diet and oral iron was terminated. Over the next few months, her symptoms resolved and her weight increased to $60 \mathrm{~kg}$. In January 2008, her hemoglobin was normal (125 g per L) and tTG IgA antibody was reduced (31 units). Duodenal biopsies were now normal. In May 2010, blood studies were normal including her hemoglobin (141 g per L), MCV (90 fl), ferritin (22 ug per L) and tTG IgA antibody (18 units).

Because of the history of past maternal anemia, her 82-yr-old Romanian-born mother was also reviewed. Her daughter was concerned so she purchased a commercial test kit for celiac disease from a local pharmacy and the result was positive. She was entirely asymptomatic and examination was normal. Blood studies were normal including her hemoglobin (129 g per L) and ferritin, but her tTG IgA was 135 units and subsequent duodenal biopsies showed severe architectural changes with crypt hyperplastic villus atrophy (i.e., Marsh 3). Other family members living in Canada were also screened (father, daughter) and both had normal tTG antibody levels (less than 20 units). Her mother was treated with a gluten-free diet.

Case 2. A 45-yr-old female, born in Czechoslovakia (now Slovakia), complained to her family physician of abdominal bloating, constipation and pale stools in February 2006. She also noted a blistering skin rash on the extensor surfaces of her limbs, likely dermatitis herpetiformis. Her physician suspected colonic blood loss because iron deficiency anemia was present (hemoglobin of $96 \mathrm{~g}$ per $\mathrm{L}$ and a low serum ferritin with normal serum folate and vitamin B12), so colonoscopic evaluation was requested. Iron treatment was initiated and she was reviewed in June 2006. Her hemoglobin showed minimal improvement to $100 \mathrm{~g}$ per L while ferritin remained severely abnormal at 4.1 ug per L. Because of her constipation, oral iron was discontinued. Colonoscopy and biopsy were normal. A tTG IgA antibody was increased (123 units). UGI endoscopy showed scalloped duodenal mucosal folds and biopsies showed severe architectural changes with crypt hyperplastic villus atrophy (i.e., Marsh 3). A gluten-free diet was initiated. On a gluten-free diet, her weight increased by 11 $\mathrm{kg}$ with normalization of her hemoglobin to $120 \mathrm{~g}$ per L. A repeat tTG assay was improved to 25 units. Duodenal biopsies now showed persistent, but improved, architectural changes with reappearance of villi and persistent intraepithelial lymphocytosis (i.e., Marsh 1-2).
Case 3. A 39-yr-old female professional dietitian was referred in July 1990 for investigation of diarrhea, 4 to 6 times daily, for 6 months. There was no abdominal pain or weight loss. About a decade earlier, she suffered from heavy menstrual bleeding, but now her menses were regular with no excess bleeding. At that time, she was reported to have anemia, received oral iron but only showed a limited hematological response, and so iron was stopped. Fecal studies for a bacterial infectious agent or a parasite were negative. Blood studies showed a mild anemia of $108 \mathrm{~g}$ per L (normal, 118 to 150 ), MCV $78 \mathrm{fL}$ (normal 82 to 97), serum iron 4.4 mol per L (normal, 6.6 to 30.4 ) and serum ferritin $<5$ ug per L (normal, 7 to 114 ). Other blood studies were normal, so she consumed oral iron. A repeat hemoglobin was $106 \mathrm{~g}$ per L. Subsequent barium studies of the upper and lower gastrointestinal tract were normal. Flexible sigmoidoscopy and biopsy were normal. UGI endoscopy showed scalloped duodenal mucosal folds and biopsies showed severe architectural changes with crypt hyperplastic villus atrophy (i.e., Marsh 3 ), however, before the latter results were formally ready, the patient initiated a strict gluten-free diet with no other treatment. In October 1990, her symptoms had resolved and repeat blood studies were normal, including hemoglobin of 135 (normal, 118-154 g per L), MCV of $89 \mathrm{fl}$ (normal, 80 to 98 ), serum iron 16 mol per L (normal, 10 to 33 ) and serum ferritin 26 ug per L (normal, 25 to 250). As she moved to another city, another biopsy could not be done.

Case 4. A 33-yr-old female university student was referred because of intermittent anorectal pain and rectal bleeding in March 2001. She had normal menses. Blood studies revealed a hemoglobin of $119 \mathrm{~g}$ per L (normal, 120 to 150) and a serum ferritin of 4 ug per L (normal 10 to 160). Serum folic acid and vitamin B12 were normal. A flexible sigmoidoscopy revealed normal colonic mucosa with an anal fissure. Local treatment led to symptomatic resolution of her pain, but a repeat hemoglobin in October 2001 was $101 \mathrm{~g}$ per L. A screening tTG IgA antibody test was positive with a value over 100 units (normal, <20), so UGI endoscopy and duodenal biopsies were done. These revealed severe architectural changes (i.e., Marsh 3) and a gluten-free diet was instituted without iron supplements. In January 2003, hemoglobin was $126 \mathrm{~g}$ per L, tTG IgA antibody was 16 units and repeat duodenal biopsies were normal.

Case 5. A 46-yr-old female was referred in May 1997 for evaluation of intermittent abdominal pain and constipation over many years. She was treated by her hematologist for anemia during pregnancy in 1992. At that time, her hemoglobin partially improved, but did not normalize, with oral iron supplements. Instead, iron seemed to worsen her constipation, so these were terminated. Pregnancy went to term, but because of persistent anemia, she was referred to exclude celiac disease. Her hemoglobin was $103 \mathrm{~g}$ per L, serum iron 3 mol per $\mathrm{L}$ and serum ferritin $4 \mathrm{ug}$ per L. An UGI endoscopy showed changes of lymphocytic gastritis while duodenal mucosal biopsies showed severe architectural changes of celiac disease (i.e., Marsh 3). Treatment consisted of a gluten-free diet. In March 1999, her hemoglobin was $134 \mathrm{~g}$ per L and serum ferritin was $16 \mathrm{ug}$ per L. A second UGI endoscopy in August 1999 was normal with normal duodenal biopsies. 
Case 6. A 78-yr-old male advertising executive was referred for evaluation of diarrhea and weight loss in March 1994. He avoided milk and dairy products, "wheat" and vegetables and supplemented his diet with concentrated carbohydrate solutions, "energy" drinks and daily multivitamins containing iron. He described his diarrhea as unpredictable, sometimes nocturnal and up to 10 per day without blood. He also noted a $10 \mathrm{~kg}$ weight loss over a month long period and attributed his symptoms to selfdiagnosed "colitis", present since 1965. However, fecal studies for bacteriology and parasites as well as a colonoscopy and biopsies were normal. Because of prior treatment with aureomycin, fecal studies for Clostridium difficile toxin were negative. His hemoglobin was $107 \mathrm{~g}$ per L (normal, 140 to 180 ), serum iron 5 umol per L (normal, 7 to 23 ) and serum albumin $24 \mathrm{~g}$ per L (normal, 35 to 50). UGI endoscopy in October 1994 was normal, but duodenal mucosal biopsies revealed severe architectural changes of untreated celiac disease (i.e., Marsh 3). Anti-gliadin antibodies were 220 units (normal, 0 to 20). A gluten-free diet was initiated. He agreed to cease use of his other self-administered treatments. His diarrhea resolved and his weight increased by $20 \mathrm{~kg}$. By September 1995, his hemoglobin was $138 \mathrm{~g}$ per L (normal, 133 to 165), serum ferritin was 47 ug per L (normal, 20 to 325) and albumin was $33 \mathrm{~g}$ per L. Anti-gliadin antibodies had normalized to 18 units. In February 2002, an UGI endoscopy was normal with normal duodenal mucosal biopsies while tTG $\operatorname{IgA}$ antibody test was normal at 4 units.

Case 7. A 32-yr-old female physical education teacher at an elite private high school was evaluated in November 1989 for chronic diarrhea, present for at least 8 months. Due to increasing fatigue, she could not continue her regular teaching duties. Her hemoglobin was 67 and so, oral iron was initiated. Fecal studies for infectious agents were negative. Repeated blood studies revealed a hypochromic microcytic anemia with a hemoglobin of 74 $\mathrm{g}$ per L and an MCV of $62 \mathrm{fl}$. Serum iron was 3 umol per $\mathrm{L}$ and serum ferritin was 8 ug per L. UGI endoscopy appeared normal but biopsies showed severe duodenal mucosal architectural disturbance (i.e., Marsh 3), typical of untreated adult celiac disease. A gluten-free diet was initiated, but she elected not to take oral iron because of taste intolerance. In January 1990, her hemoglobin was $126 \mathrm{~g}$ per L and serum iron was $9 \mathrm{~mol}$ per L. Over the next 2 months, her diarrhea had resolved and weight increased. In March 1990, her hemoglobin was 137 g per $\mathrm{L}$, serum iron was $9 \mathrm{~mol}$ per $\mathrm{L}$ and serum ferritin was 16 ug per L. Repeat biopsies showed mild inflammatory changes only with increased intra-epithelial lymphocytes (i.e., Marsh 1). She was able to return to teaching and last reviewed in 2007 at the age of 50 years. Investigations were normal, including an UGI endoscopy, duodenal mucosal biopsies and a screening colonoscopy.

Case 8. A 27-yr-old female journalism student and marathon runner was evaluated in December 2010 for profound fatigue associated with anemia. Initial studies revealed a hemoglobin of $72 \mathrm{~g}$ per L and an MCV of $74 \mathrm{fl}$. Although transfusions were considered, her physician elected to treat her with a calculated intravenous iron dextran infusion dose thought to be sufficient to increase her hemoglobin to normal with a dose of $1000 \mathrm{mg}$ of elemental iron. Other studies showed an elevated tTG IgA antibody of 45.1 units. Approximately 2 weeks later, her hemoglobin had only risen to $105 \mathrm{~g}$ per $\mathrm{L}, \mathrm{MCV}$ was $84 \mathrm{fl}$ and ferritin was 4 ug per L. A further iron dextran infusion of $1000 \mathrm{mg}$ elemental iron was electively administered in spite of the suboptimal response. Further blood studies in March 2011 revealed her hemoglobin was still low at $98 \mathrm{~g}$ per L, MCV $83 \mathrm{fL}$, serum iron $5 \mathrm{~mol}$ per L and serum ferritin 2 ug per L. UGI endoscopy was normal but duodenal mucosal biopsies were severely abnormal with absence of villi and crypt hyperplasia (i.e., Marsh 3). She was treated with a gluten-free diet. In May 2011, her hemoglobin was now $129 \mathrm{~g}$ per L, MCV $85 \mathrm{fL}$, serum ferritin 27 ug per L and tTG was 8.1 units. A computerized tomographic study with contrast of her small intestine was normal. No abnormalities were detected in her colon. Another small intestinal biopsy could not be repeated.

\section{Discussion}

Iron deficiency anemia is a common clinical presentation of celiac disease with or without other symptoms (eg., diarrhea, weight loss) and a number of possible mechanisms for iron deficiency have been suggested [1]. For example, impaired duodenal mucosal uptake of iron may occur related to the presence of abnormal duodenal mucosal cells and reduced surface absorptive area. In addition, some may have microscopic blood loss from celiac-associated mucosal erosions or ulceration, or alternatively, an ulcerating complication of celiac disease (eg., malignancy). During pregnancy, fetal iron requirements may add further demands on normally-present or borderline maternal iron stores. Autoimmune intravascular hemolysis may also complicate celiac disease resulting in urinary iron loss [2]. Other rare causes of anemia, including sideroblastic anemia have been described that may respond to a glutenfree diet [3]. Finally, in some, and particularly intriguing, erythropoiesis may be further suppressed because of the presence of a persistent and chronic intestinal or associated extra-intestinal inflammatory process that ultimately, may be reversed with a gluten-free diet.

After initial detection, most clinical features of celiac disease fortunately resolve with a strict gluten-free diet, including the anemia. In some adults with newly diagnosed celiac disease, iron supplements may also be administered. However, the present series of patients reported here emphasize that the key element in treatment of the anemia in these patients was the gluten-free diet per se. In all, iron administered to treat the anemia was initially ineffective in improving the hematological state, even after many months of iron supplements. Similar observations were made in a previous report of 2 celiacs that showed no improvement in iron levels and anemia with oral iron supplements alone until a strict gluten-free diet was instituted [4]. Here, a strict gluten-free diet alone was sufficient to actually treat the anemia per se, especially if iron supplements were poorly tolerated. Finally, in one case, sufficient elemental iron administered in intravenous form also provided only a partial hematological response, until a strict gluten-free diet was administered. In this case, resolution of anemia was 
anticipated after a calculated dose of elemental iron (and then a similar second dose), but anemia failed to resolve. Although the precise reason is not known, previous reports have suggested that other causes for anemia, including the "anemia of chronic disease", may be present in celiac disease in addition to iron and vitamin deficiencies [5]. Added studies are needed to determine if the presence of an ongoing chronic intestinal inflammatory process impairs normal erythropoiesis or some other iron homeostatic regulatory control mechanism in untreated celiac disease that is corrected with a gluten-free diet.

Failure to respond to oral iron with iron deficiency anemia usually results in further studies to determine the cause. Investigations are often focused on a cause for persistent or ongoing blood loss, often from the gastrointestinal tract (eg., occult colonic cancer). In the present selected series of adults, however, iron deficiency anemia that failed to respond to iron administration was later shown to be related to occult and previously unrecognized celiac disease. Once dietary treatment with a strict gluten-free diet was initiated leading to resolution of the underlying intestinal disease, iron deficiency anemia also resolved, even without added iron supplements. Iron refractory or resistant iron deficiency anemia (in contrast to iron deficiency anemia alone) has been underemphasized as a possible presentation of underlying adult celiac disease [6]. In children, other causes for iron refractory or iron resistant iron deficiency anemia may occur. For example, an unusual and rare inherited recessive gene defect central to control of a regulatory protein involved in iron homeostasis may be altered (eg., TMPRSS6 gene to encode matriptase-2, a transmembrane serine protease known to down-regulate hepcidin) $[7,8]$. Future studies in adults may lead to detection of other defective genes involved in regulation of iron homeostasis.

\section{References}

[1] Freeman HJ. Iron deficiency anemia in celiac disease. World J Gastroenterol 2015; 21: 9233-9238.

[2] Miller DG. Celiac disease with autoimmune hemolytic anemia. Postgrad Med J 1984; 60: 629-630.

[3] Dawson AM, Holdsworth CD, Pitcher CS. Sideroblastic anemia in adult celiac disease. Gut 1964; 5: 304-308.

[4] Sari R, Yildirim B, Sevince A, Buyukberber S. Gluten-free diet improves iron-deficiency anemia in patients with celiac disease. J Health Popul Nutr 2000; 18: 54-56.

[5] Bergamaschi G, Markopoulos K, Albertini R, Di Sabatino A, Biagi F, Ciccocioppo R, Arbustini E, Corazza GR. Anemia of chronic disease and defective erythropoietin production in patients with celiac disease. Haematologica 2008; 93: 1785-1791.

[6] Annibale B, Severi C, Chistolini A, Antonelli G, Lahner E, Marcheggiano A, Iannoni C, Monarca B, Delle Fave G. Efficacy of gluten-free diet alone on recovery from iron deficiency anemia in adult celiac patients. Am J Gastroenterol 2001; 96: 132-137.

[7] Finberg KE. Iron-refractory iron deficiency anemia. Semin Hematol 2009; 46: 378-386.

[8] Cau M, Melis MA, Congiu R, Galanello R. Iron-deficiency anemia secondary to mutations in genes controlling hepcidin. Expert Rev Hematol 2010; 3: 205-216. 